\title{
What Could It Be? Gluten-Sensitive Enteropathy
}

\section{Kristy O. Oden}

Anderson College of Nursing, University of North Alabama, Florence, AL 35632, USA

\section{Abstract}

What do I do? A 34-year-old female patient presents to the gastrointestinal office with the following complaints: joint pain, headaches, mild anemia, dry mouth, anxiety, and fatigue. Occasionally she has abdominal discomfort and bloating. These certainly do not sound like symptoms that are typical in a gastrointestinal office, but they could be.

\section{Publication History:}

Received: August 09, 2016

Accepted: October 15, 2016

Published: October 17, 2016

\section{Keywords:}

Gluten sensitive Enteropathy, Celiac disease, Autoimmune, Diabetes

\section{Gluten-Sensitive Enteropathy: Facts}

According to the Celiac Support Association [1] and the University of Chicago Celiac Disease Center [2] Celiac Disease has the following facts:

- It is estimated that more than 3 million individuals in the US are affected by Celiac Disease but approximately $1 / 2$ are undiagnosed

- Over $1 / 2$ of those diagnosed with Celiac Disease were asymptomatic

- Initial diagnosis of Celiac Disease can take between 4-6 years but up to greater than 10 years

- Individuals, who have a parent, child, or sibling with Celiac Disease, have a 1 in 22 chance of a diagnosis of Celiac Disease

- Individuals, with an aunt, uncle, or cousin diagnosed with Celiac Disease, also have an increased risk of a diagnosis of Celiac Disease

- Though there is a family connection, the inheritance pattern is unknown

- Healthcare costs in the US for those undiagnosed can exceed $\$ 30$ billion dollars

\section{Gluten-Sensitive Enteropathy: Description}

Gluten-Sensitive Enteropathy, also known as Celiac Disease, is an autoimmune disease. This disease focuses on the small bowel and requires lifelong treatment [3]. It is genetically linked, and according to the U.S. National Institute of Health, there are about 1 in 100 people worldwide that are affected by it. When anindividual consumes the gluten protein that is naturally found in wheat, barley, and rye the disease is triggered (National Institute of Diabetes and Digestive and Kidney Diseases [4]. These items are frequently found in pastas, breads, and bakery products but can also be found in unexpected locations such as pre-packaged lip balms, hair and skin products, and many other items used on a daily basis [4].

If an individual has a diagnosis of Type 1 Diabetes Mellitus, Turner Syndrome, Down Syndrome and other diseases, they may be at an increased risk for Celiac Disease [5]. Additionally, the disease can be difficult to diagnose because symptoms mimic those of inflammatory bowel disease and irritable bowel disease [6]. Gleaning knowledge about Gluten-Sensitive Enteropathy will allow healthcare providers to diagnose, treat, and manage the disease thus improving patient outcomes.

\section{Gluten-Sensitive Enteropathy: Pathophysiology}

When discussing the pathophysiology of Celiac Disease, one must first define autoimmune disease. Autoimmune disease occurs when the body, specifically the immune system, reacts against self-antigens. This reaction leads to damage to that individuals own tissue due to autoantibiodies [3].

Three issues, genetics, immunity, and environment, must be considered when discussing Celiac Disease. The environmental factor is the ingestion of gluten through a variety of products. Genetically, there is the presence of an antigen identified in the blood. The intestinal cells become injured through a T-cell-mediated autoimmune response [3]. "Transglutaminase 2 (TG2) and endoymsial autoantibodies closely correlate with the acute phase of the disease in the presence of gluten. T-cell infiltration results in mucosal cell destruction with inflammation, atrophy, and flattening of villi in the upper small intestine" [3]. Thus, cell death exceeds cell reproduction and the immature cells cannot sustain proper absorptive functions, particularly in the duodenum and jejunum [3]. The damage leads to malabsorption.

\section{Gluten-Sensitive Enteropathy: Symptoms}

As we have already identified, more than 3 million individuals in the US are affected by Celiac Disease, yet only one-half of those have been diagnosed. The high rate of delayed or no diagnosis can be attributed to the symptoms, which vary depending on age. Since this is considered a gastrointestinal disease, an expectation would be gastrointestinal symptoms, however this is not always the case. Digestive symptoms can be present, but are more typical in children. Absorption of nutrients is very important for the growth and development of children, however, if Celiac Disease is present, that child is not absorbing essential nutrients. Commonly, in children gastrointestinal symptoms include $[7,8]$ :

$$
\begin{aligned}
& \text { - } \quad \text { Abdominal pain and bloating } \\
& \text { - } \quad \text { Diarrhea and/or constipation } \\
& \text { - } \quad \text { Pale, foul-smelling stool } \\
& \text { - } \quad \text { Fatty, floating stool }
\end{aligned}
$$

"Corresponding Author: Dr. Kristy O. Oden, Anderson College of Nursing University of North Alabama, Florence, Alabama, AL 35632, USA; E-mail: kooden@una.edu

Citation: Oden KO (2016) What Could It Be? Gluten-Sensitive Enteropathy. Int J Nurs Clin Pract 3: 201. doi: http://dx.doi.org/10.15344/2394-4978/2016/201

Copyright: (c) 2016 Oden. This is an open-access article distributed under the terms of the Creative Commons Attribution License, which permits unrestricted use, distribution, and reproduction in any medium, provided the original author and source are credited. 


- $\quad$ Nausea and/or vomiting
- $\quad$ Delayed growth/failure to thrive
- $\quad$ Fatigue
Adults, however, are less likely to exhibit the same symptoms, in
fact, they may not have digestive symptoms. They have may some of
the following symptoms [7,8]:
- $\quad$ Anemia, usually unexplained iron-deficiency anemia
- Smooth, red, shiny tongue
- Joint pain
- Osteopenia and/or osteoporosis
- Changes in mood, i.e. depression, anxiety
- Dermatitis herpetiformis: itchy, blistering rash most commonly
- $\quad$ found on the knees, elbows, scalp, and back
- $\quad$ Headaches and/or migraines
- Dry mouth
- $\quad$ Canker sores in the mouth
- $\quad$ Fatigue

Digestive symptoms may be exacerbated as abdominal pain with bloating, blockages within the intestines, chronic fatigue, and sores or ulcers found in the lining of the intestine [8]. Immune reactions outside of the digestive system can occur as a result of Celiac Disease being autoimmune. Liver and biliary tract disorders can occur, as well as skin, nervous system and skeletal system disorders $[7,8]$ ).

Symptoms vary among individuals and even those who are asymptomatic are at risk for developing complications over time. Symptoms may be affected by how much gluten is consumed, age of first exposure to gluten (i.e. the longer an infant is breast-fed, the later the diagnosis), and the amount of damage to the lining of the small intestines [8]. No matter the symptoms, or lack thereof, Celiac Disease only affects those who have specific genes and that are consuming foods containing gluten [8].

\section{Gluten-Sensitive Enteropathy: Diagnosis}

So many individuals go undiagnosed for so long, because only a small portion of the intestine is affected or the inflammation is very mild [9]. Since so many of the symptoms of Celiac Disease mimic other disease processes, differentiation is difficult. Individuals initial presentations may be so mild or so basic, that the healthcare provider initially assumes that it may be vial or bacterial in nature, irritable bowel syndrome (IBS), inflammatory bowel disease (IBD), or some type of nutritional imbalance [10]. In individuals who have opted to eliminate gluten in their diet prior to testing, they may receive a delayed diagnosis due to false laboratory results or inaccurate endoscopic biopsies [10]. Since celiac disease has autoimmune featuresand relations, those who are already on immunosuppressant therapy could also have delayed diagnosis [10]. In individuals whom the healthcare provider strongly suspects gluten-sensitive enteropathy and who has negative results on initial testing, recommendations are to ensure that the individual has not removed gluten from his/her diet, review medications that could lead to false test results, and repeat testing.
Healthcare provider will include family history along with a physical examination where the body will be evaluated for rashes and malnutrition, and a thorough abdominal examination will be conducted 8]. Recommendations for a diagnosis indicate that if four out of the following five conditions are present, then a diagnosis of Celiac Disease should be established [9]:

\section{Typical symptoms present \\ 2. Positive serum lab work \\ 3. DQ2 or HLA-DQ8 genotype \\ 4. Small-bowel biopsy shows celiac enteropathy \\ 5. Gluten-free diet had a positive response}

Serum lab work, though beneficial, should not be used alone for diagnostic purposes, as they are not always conclusive. The antitTG (anti-tissue transglutaminase antibody) is the most specific and sensitive test [11] along with the antiendomysial antibody (EMA) test [9]. Intestinal biopsy, with confirmation of celiac enteropathy, is considered the gold standard [9]. Knowing that dermatosis herpetiformis is associated with Celiac Disease, a skin biopsy can be utilized to confirm this. Alternate literature indicates that positive antibody testing, along with a positive skin biopsy can eliminate the need for an intestinal biopsy $[5,6]$. If biopsies and serum laboratory evaluations are inconclusive, genetic testing can be considered [8].

\section{Gluten-Sensitive Enteropathy: Treatment}

Treatment is challenging for the patient and the healthcare provider. Primary treatment is a gluten-free diet. One note to remember is that once an individual is placed on a gluten free diet for approximately one year, his/her repeat endoscopic biopsies will typically be negative for gluten-sensitivity enteropathy, as there is no further inflammation. For those that are asymptomatic at diagnosis, adherence to a gluten-free diet seems extreme. Education is the key! Healthcare providers must encourage these patients to adhere to this diet, acknowledging that complications such as other auto-immune diseases, long- term malnutrition, osteoporosis, and reproductive and nervous system problems are associated complications of Celiac Disease [8]. Rarely, intestinal cancer, lymphoma, and liver disease can arise as a complication of Celiac Disease [8]. With lack of gluten in the diet, the intestines are allowed to heal, leading to symptom reduction. Although some improvement in symptoms may occur after just a few days of dietary change, complete healing can take weeks and even years.

Since Celiac Disease is associated with malabsorption and nutritional deficiencies, dietary supplements may be needed. Iron deficiency anemia is commonly present in individuals with Celiac Disease as well as Folic Acid, Vitamin B12 and D deficiency. Supplementation of these vitamins could help reduce neurologic and bone complications [9].

Individuals who consume a gluten-free diet may have diarrhea related to a compromised digestive process. These individuals may benefit from ingestion of oral pancreatic enzymes [9]. Additionally, probiotics may have a positive outcome on inhibiting damage to the intestinal epithelial caused by altered intestinal bacterial composition [9]. 


\section{Gluten-Sensitive Enteropathy: Education}

Education is key and once diagnosed, individuals with Celiac Disease are asked to make major life changes. Healthcare providers instruct patients to eat a gluten-free diet, but what does that mean?

Gluten is a protein. It is found naturally in wheat, barley, and rye [8]. Gluten is also hidden in food substances and other products [6]. According to O’Donnell [6], "Gluten is used as a stabilizer, emulsifier, and thickening agent in a vast amount of processed foods" (pg. 5). Gluten may be found in herbal/nutritional supplements, medications, children's modeling dough, cosmetics and beauty products, and healthcare products [8]. A referral to a dietician would be beneficial in helping individuals to learn to read labels, plan meals, and make healthy choices [8]. Educational resources include, but are not limited to, Celiac Disease Foundation, www.cealiac.org, Celiac Support Association, www.csaceliacs.org, and www.celiac.com. Many of these websites offer lists of gluten free products and recipes, as well as support groups. Remember, one can never have too much knowledge!

\section{Competing Interests}

The author have no conflict of interests to disclose.

\section{Author Contributions}

Dr. Oden has been a Family Nurse Practitioner, working in the area of Gastroenterology for the past 11 years and has been in full time education for the past 6 years. She has authored or co-authored articles on Barrett's Esophagus and Transitioning Nurse Practitioners into Associate Professors. Her doctoral research focused on community awareness of colorectal cancer. She has presented at the local, state, and international levels. Most recently, she received the Phi Kappa Phi Honor Society Eleanor Gaunder Teaching Excellence Award, a peer nominated award.

\section{Acknowledgements}

Thanks goes out to my family, who continue to support me, and to the Anderson College of Nursing for continuing to challenge the faculty.

\section{References}

1. Celiac Support Association (2016) Celiac Disease Facts.

2. University of Chicago Celiac Disease Center (2016) Celiac Disease Facts and Figures.

3. McCance KL, Huether SE (2014) Pathophysiology, (7th Edition).

4. National Institute of Diabetes and Digestive and Kidney Diseases (NIDDK) (2016).

5. National Institute of Diabetes and Digestive and Kidney Diseases (NIDDK) (2016). Definition and Facts for Celiac Disease.

6. O'Donnell L (2016) Understanding Celiac Disease: A Recipe for Nursing Care. Med-Surg Matters 25: 4-6.

7. Celiac Disease Foundation (2016) Celiac Disease Symptoms.

8. National Institute of Diabetes and Digestive and Kidney Diseases (NIDDK) (2016) Symptomsand Causes of Celiac Disease.

9. Horowitz S (2011) Celiac Disease: New Directions in Diagnosis, Treatment, and Prevention. Alternative and Complementary Therapies 17: 92-98.

10. Pelkowski T, Viera A (2014) Celiac Disease: Diagnosis and Management. American Family Physician 89: 99-105.

11. Talaie $R$ (2015) Does gluten free diet have more implications than treatment of celiac disease? Gastroenterol Hepatol Bed Bench 8: 160-165. 